\title{
Influence of surface defects on vortex penetration and expulsion in mesoscopic superconductors
}

\author{
B. J. Baelus* \\ Departement Fysica, Universiteit Antwerpen (Campus Drie Eiken), Universiteitsplein 1, B-2610 Antwerpen, Belgium and \\ Institute of Materials Science, University of Tsukuba, Tsukuba 305-8573, Japan \\ K. Kadowaki ${ }^{\dagger}$ \\ Institute of Materials Science, University of Tsukuba, Tsukuba 305-8573, Japan \\ F. M. Peeters \\ Departement Fysica, Universiteit Antwerpen (Campus Drie Eiken), Universiteitsplein 1, B-2610 Antwerpen, Belgium
}

(Received 10 September 2004; published 25 January 2005)

\begin{abstract}
Solving the nonlinear Ginzburg-Landau equations self-consistently we investigated the influence of surface defects on the vortex penetration and expulsion in thin mesoscopic superconducting samples. The effect of the number, size, and position of surface defects on the vortex entry and exit fields and on the entrance and exit positions of the vortex are studied for very thin circular, square, and rectangular samples. For specific vortex configurations we found that due to the interplay between the vortex-vortex repulsion and the vortex-defect interaction, the vortex does not enter or leave the sample through the surface defect.
\end{abstract}

DOI: 10.1103/PhysRevB.71.024514

PACS number(s): 74.20.De, 74.25.Dw, 74.25.Ha

\section{INTRODUCTION}

In the last decade, the study of vortex matter in finite mesoscopic superconductors has attracted a lot of attention, both theoretically and experimentally (see, e.g., Refs. 1-7). It became generally known that metastable vortex states can nucleate, which show up as an hysteresis effect at the transitions between different vortex states, i.e., during the penetration and expulsion of vortices. This hysteresis effect is due to the presence of a surface barrier. ${ }^{8}$ Theoretically, one often considered perfect smooth samples, i.e., with no surface defects, which leads to a maximum surface barrier and maximum hysteresis effect. ${ }^{9}$ On the other hand, it is very difficult to experimentally create a superconductor with a perfectly smooth boundary and one should deal theoretically with defects at the surface. These surface defects can act as nucleation centers for vortex entry and exit, which decrease the surface barrier. Therefore, we expect that the presence of surface defects leads to higher expulsion fields and lower penetration fields, and thus to a reduction of the hysteresis effect and a reduction of the magnetic field region over which the metastable states are stable. For this reason, a theoretical study of the effect of surface defects on vortex penetration and expulsion is very important for a comparison of the theoretical and experimental results.

During the last few years several theoretical studies were performed where the influence of surface defects on the vortex entry in bulk superconductors was investigated. ${ }^{10-13}$ In bulk superconductors it is indeed found that the presence of a surface defect leads to lower penetration fields and that the stability region of the metastable states decreases.

Surface defects will play an even more pronounced role in mesoscopic superconductors, due to the small size of their boundary. In the present paper, we will investigate the effect of surface defects in mesoscopic superconductors. We will determine the effect of the number of surface defects, the position of the surface defect, and the size of the surface defect on the vortex expulsion and penetration. The path of the entering (or exiting) vortex with respect to the position of the defect will be investigated for different shapes of the superconducting sample.

We consider thin superconductors immersed in an insulating medium in the presence of a perpendicular uniform magnetic field $H_{0}$. To solve this problem we follow the numerical approach of Schweigert and Peeters. ${ }^{3,4}$ As for thin disks $(d$ $\ll \xi, \lambda)$ averaging the GL equations over the disk thickness is allowed. Using dimensionless variables and the London gauge $\operatorname{div} \vec{A}=0$ for the vector potential $\vec{A}$, we write the system of GL equations in the following form:

$$
\begin{gathered}
\left(-i \vec{\nabla}_{2 \mathrm{D}}-\vec{A}\right)^{2} \Psi=\Psi\left(1-|\Psi|^{2}\right) \\
-\Delta_{3 \mathrm{D}} \vec{A}=\frac{d}{\kappa^{2}} \delta(z) \vec{j}_{2 \mathrm{D}}
\end{gathered}
$$

where

$$
\vec{j}_{2 \mathrm{D}}=\frac{1}{2 i}\left(\Psi^{*} \vec{\nabla}_{2 \mathrm{D}} \Psi-\Psi \vec{\nabla}_{2 \mathrm{D}} \Psi^{*}\right)-|\Psi|^{2} \vec{A}
$$

is the density of superconducting current. The superconducting wave function satisfies the boundary conditions $\left(-i \vec{\nabla}_{2 \mathrm{D}}\right.$ $-\vec{A})\left.\Psi\right|_{n}=0$ normal to the sample surface and $\vec{A}$ $=1 / 2 H_{0} \rho \vec{e}_{\phi}$ far away from the superconductor. Here the distance is measured in units of the coherence length $\xi$, the vector potential in $c \hbar / 2 e \xi$, and the magnetic field in $H_{c 2}$ $=c \hbar / 2 e \xi^{2}=\kappa \sqrt{2} H_{c}$. The superconductor is placed in the $(x, y)$ plane, the external magnetic field is directed along the $z$ axis, and the indices 2D, 3D refer to two- and three-dimensional operators, respectively.

For a more detailed discussion of the self-consistent solution of Eqs. (1) and (2), we refer to Refs. 4 and 14. The dimensionless Gibbs free energies (measured in units of $F_{0}$ 

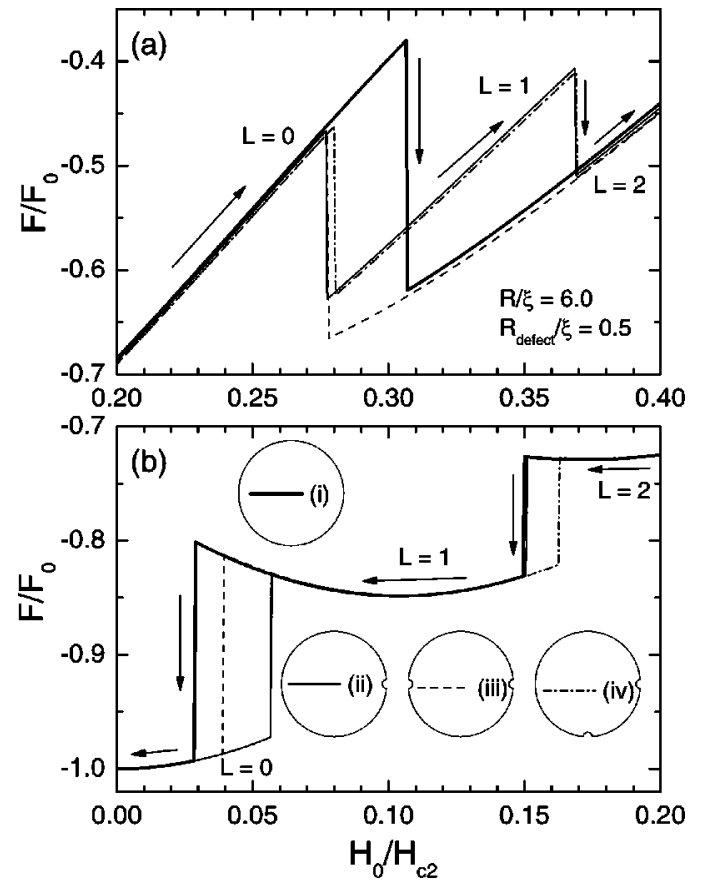

FIG. 1. The free energy as a function of (a) increasing and (b) decreasing applied magnetic field for a disk with radius $R=6.0 \xi$ with (i) no surface defects, (ii) one surface defect with radius $R_{\text {defect }}=0.5 \xi$, and (iii), (iv) two surface defects. The insets show the samples in more detail.

$\left.=H_{c}^{2} V / 8 \pi\right)$ of the different vortex configurations is given by

$$
F=V^{-1} \int_{V}\left[2\left(\vec{A}-\vec{A}_{0}\right) \cdot \vec{j}_{2 \mathrm{D}}-|\Psi|^{4}\right] d \vec{r},
$$

where integration is performed over the sample volume $V$ and $\vec{A}_{0}$ is the vector potential of the uniform magnetic field.

\section{RESULTS}

First we will concentrate on extremely thin superconductors, such that only the first GL equation [Eq. (1)] has to be solved and the magnetic field can be assumed to be homogeneous and equal to the external field $H_{0}$. Later, we will consider superconductors with finite thickness and investigate the influence of such nonzero thickness on the vortex penetration and expulsion in the presence of a surface defect including effects due to $H \neq H_{0}$.

\section{A. The effect of the number of surface defects and their relative position for a superconducting disk}

Figures 1(a) and 1(b) show the free energy as a function of increasing and decreasing applied magnetic field for a superconducting disk with no surface defects [sample (i)] by thick solid curves and the same disk with different configurations of surface defects [samples (ii)-(iv)] by thin curves. The disk has radius $R=6.0 \xi$, the circular surface defects have radius $R_{\text {defect }}=0.5 \xi$ and are centered at the surface boundary. The samples are shown in the insets. With increasing field the change in vorticity at the first transition depends on the number and positions of the surface defects [see Fig. 1(a)]. In a "perfect disk," two vortices enter when the Meissner state becomes unstable with increasing field. This can be seen from the thick curve in Fig. 1(a), which shows that the vortex state changes from the Meissner state with vorticity $L=0$ to a vortex state with vorticity $L=2$ at $H_{0} / H_{c 2}=0.307$ with increasing field. The two vortices will just enter opposite to each other, because then the distance between the two vortices is maximum, and, thus, the repulsion is minimum. When we add a surface defect [sample (ii)], the surface barrier decreases at the defect position, which leads to an earlier penetration of one vortex and a decrease of the penetration field to $H_{0} / H_{c 2}=0.227$ [see the thin solid curve in Fig. 1(a)]. Adding a second defect [samples (iii) and (iv)] reduces the penetration field to $H_{0} / H_{c 2}=0.278$ for sample (iii) and $H_{0} / H_{c 2}=0.280$ for sample (iv). However, depending on the position of the second defect, the number of vortices penetrating the sample at the first transition can vary. When the two defects are opposite from each other [sample (iii)], two vortices enter at the first penetration field [see the dashed curve in Fig. 1(a)], while for sample (iv) only one vortex enters. Notice that the penetration field is practically the same for samples (ii)-(iv), even for sample (iii) where two vortices enter at once. With decreasing field the vortices leave the sample one by one [see Fig. 1(b)] for all sample configurations. Notice that the presence of one or more surface defects enhances the expulsion field, i.e., decreases the hysteresis effect, with decreasing field. For example, the $L$ $=1 \rightarrow 0$ transition occurs at $H_{0} / H_{c 2}=0.029$ for the perfect sample (i), at $H_{0} / H_{c 2}=0.057$ for sample (ii) with one surface defect and at $H_{0} / H_{c 2}=0.039$ and $H_{0} / H_{c 2}=0.057$ for samples (iii) and (iv). Notice that for the ideal sample and for sample (iii) no two vortices are expelled at the same time.

Next, we will focus on the position where the vortices enter or leave the sample during the transitions. When no surface defects are present, there is no favorite position for the vortex to enter or leave the superconductor (see, e.g., Ref. 15). In the case of increasing field the two vortices enter opposite from each other during the first transition $(L=0$ $\rightarrow 2$ ). How is the penetration/expulsion position influenced by the presence of one or more defects?

Figures 2(a)-2(d) show the free energy evolution during the $L=0 \rightarrow 1$, the $L=1 \rightarrow 2$, the $L=1 \rightarrow 0$, and the $L=2 \rightarrow 1$ transition, respectively, for sample (ii) of Fig. 1, i.e., a disk with radius $R=6.0 \xi$ with one surface defect with radius $R_{\text {defect }}=0.5 \xi$. The insets show the Cooper-pair density of the vortex state. Inset (i) is the metastable vortex state just before the transition, inset (iv) the metastable vortex state just after the transition. Insets (ii) and (iii) are the vortex states during the transition at the iteration steps indicated by the symbols in the main figure. These insets indicate where the vortex enters or leaves the sample. Notice that the surface defect is given by the black half dot in the figures.

With increasing field, the first vortex enters through the defect [see Fig. 2(a)]. Just before the transition, the Cooperpair density has clearly the lowest value around the defect [inset (i)]. Inset (ii) shows the penetration of the vortex through the defect. After the penetration this vortex moves towards the center of the disk [inset (iii)]. Due to the presence of the defect, the stability position of the vortex is not 

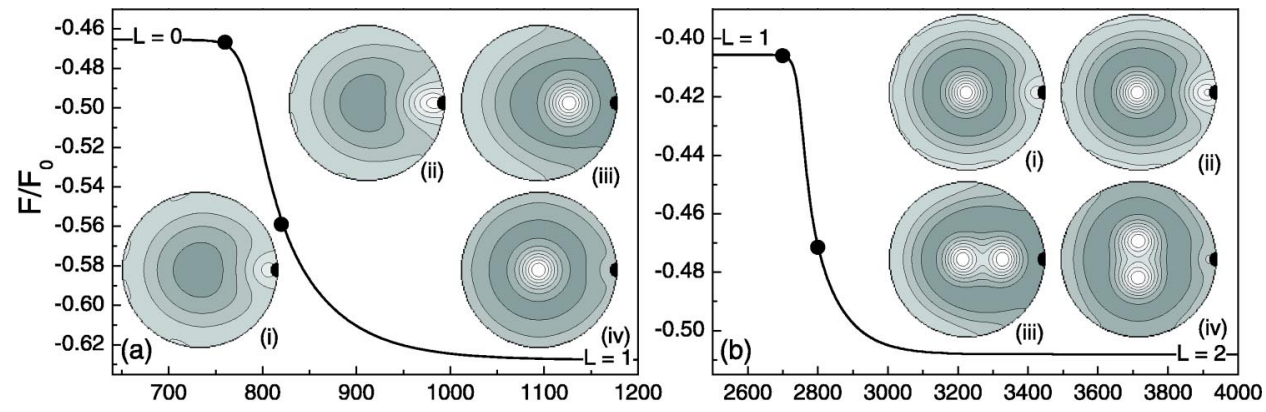
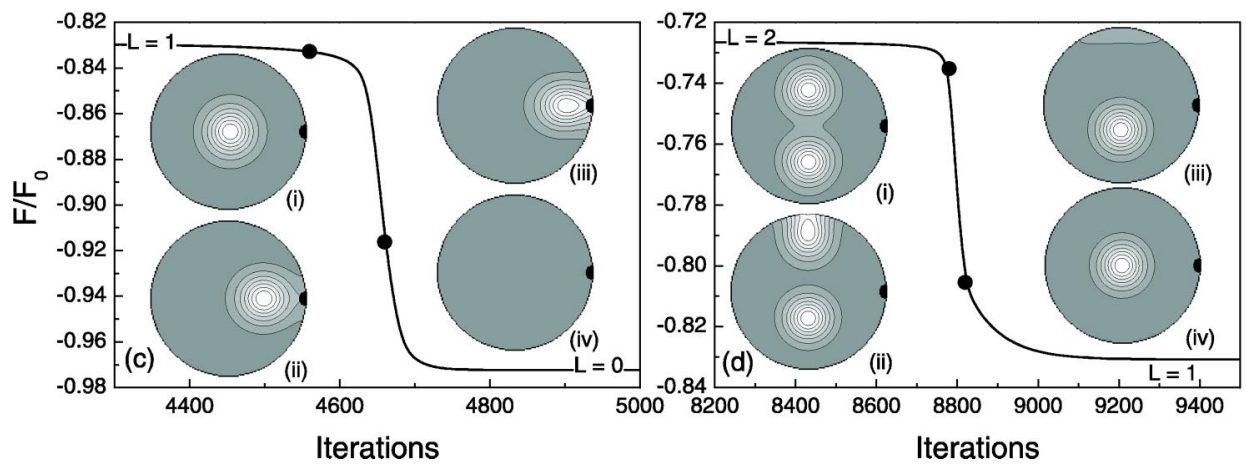

FIG. 2. The free energy evolution during (a) the $L=0 \rightarrow 1$, (b) the $L=1 \rightarrow 2$, (c) the $L=1 \rightarrow 0$, and (d) the $L=2 \rightarrow 1$ transition, respectively, for sample (ii) of Fig. 1. Inset (i) is the Cooper-pair density of the metastable vortex state just before the transition, inset (iv) the one of the metastable vortex state just after the transition. Insets (ii) and (iii) are the vortex states during the transition at the iteration steps indicated by the symbols in the main figure. High (low) Cooper-pair density is given by dark (white) regions. exactly at the center but a little bit shifted in the direction opposite to the defect, as can be seen from inset (iv). The second vortex also enters through the defect [see Fig. 2(b)]. Due to the repulsion with the penetrating vortex, the vortex in the center of the disk moves first a little bit towards the disk boundary in the opposite direction of the penetrating vortex [insets (ii), (iii)]. Finally, the state stabilizes when the two vortices are rotated over $\pi / 2$, such that they are along the diameter perpendicular to the defect [inset (iv)]. Notice that the $L=2$ state is a multivortex state at magnetic fields, just above the expulsion field. As for the case of a disk with no surface defect, the two vortices will move towards the center with increasing field and finally they combine into a giant vortex. ${ }^{15}$ The main difference due to the defect is that the degeneracy decreases. In the case of a perfect disk, the multivortex state with $L=2$ is infinitely degenerated (i.e., with respect to the rotation of the multivortex), while for the disk with one surface defect the vortex configuration is locked by the defect and the degeneracy is lifted.

With decreasing field, the last vortex leaves the sample at $H_{0} / H_{c 2}=0.057$ through the defect as shown in Fig. 2(c). Just before the transition the vortex is almost at the disk center [inset (i)], then it moves towards the defect [inset (ii)] before it leaves the sample through this defect [inset (iii)]. Finally, the state stabilizes again and the Meissner state is found [inset (iv)]. The $L=2 \rightarrow 1$ transition is more interesting [see Fig. 2(d)]. Just before the transitions, the two vortices are very close to the disk boundary [inset (i)]. Instead of rotating over $\pi / 2$, such that one vortex can leave through the defect, the vortex molecule stays along the same direction and the vortex leaves the sample not through the defect [inset (ii)]. This explains why the $L=2 \rightarrow 1$ expulsion field is the same as for the sample without defects. After the expulsion, the remaining vortex moves towards the center [inset (iii)] and finally the stable $L=1$ state is found [inset (iv)].

Next, we study the vortex penetration and expulsion when two surface defects are present. To reduce the amount of figures, we will restrict our discussion to the contour plots, which clearly show where the vortices enter or leave the sample during the iteration process. Figures 3(a)-3(c) show those contour plots of the Cooper-pair density for the $L=0$ $\rightarrow 2$, the $L=2 \rightarrow 1$, and the $L=1 \rightarrow 0$ transition in a superconducting disk with radius $R=6.0 \xi$ with two surface defects with radius $R_{\text {defect }}=0.5 \xi$, which are located opposite to each other [sample (iii) of Fig. 1]. With increasing field two vortices enter at the first transition field (see also Fig. 1). Figure 3(a) shows that the penetration of the two vortices occurs simultaneously through both defects. After the penetration the two-vortex molecule rotates over $\pi / 2$ as was observed in Fig. 2(b). On the other hand, the expulsion of the vortices is one by one, but the vortices do not always leave the sample through one of the defects. The $L=2 \rightarrow 1$ transition is very similar to the one for the one defect situation, which explains why their expulsion fields are the same. The vortices do not rotate first so that the expulsion is not through a defect [see Fig. 3(b)]. The last vortex leaves the sample through one of the defects with decreasing field [see Fig. 3(c)]. Figures $3(d)-3(g)$ show the contour plots of the Cooper-pair density for the $L=0 \rightarrow 1$, the $L=1 \rightarrow 2$, the $L=2 \rightarrow 1$, and the $L=1$ $\rightarrow 0$ transition in sample (iv) of Fig. 1. At the first transition, the first vortex enters the disk through one of the defects with increasing field [see Fig. 3(d)]. Also the second vortex enters the disk through one of the defects [Fig. 3(e)]. After the vortex entry, the two vortices rotate over $-\pi / 4$ and the twovortex state stabilizes. With decreasing field, the vortex molecule rotates over $\pi / 4$ during the $L=2 \rightarrow 1$ transition and the last but one vortex leaves the sample through one of the defects [Fig. 3(f)]. Also the last vortex leaves the superconductor through one of the two defects [see Fig. 3(g)].

So far, we have studied the influence of one or more defects on the transition fields and the position of the vortex entry and exit in a superconducting disk with radius $R$ $=6.0 \xi$ with defects with radius $R_{\text {defect }}=0.5 \xi$. We found that the hysteresis effect decreases, i.e., the penetration field de- 

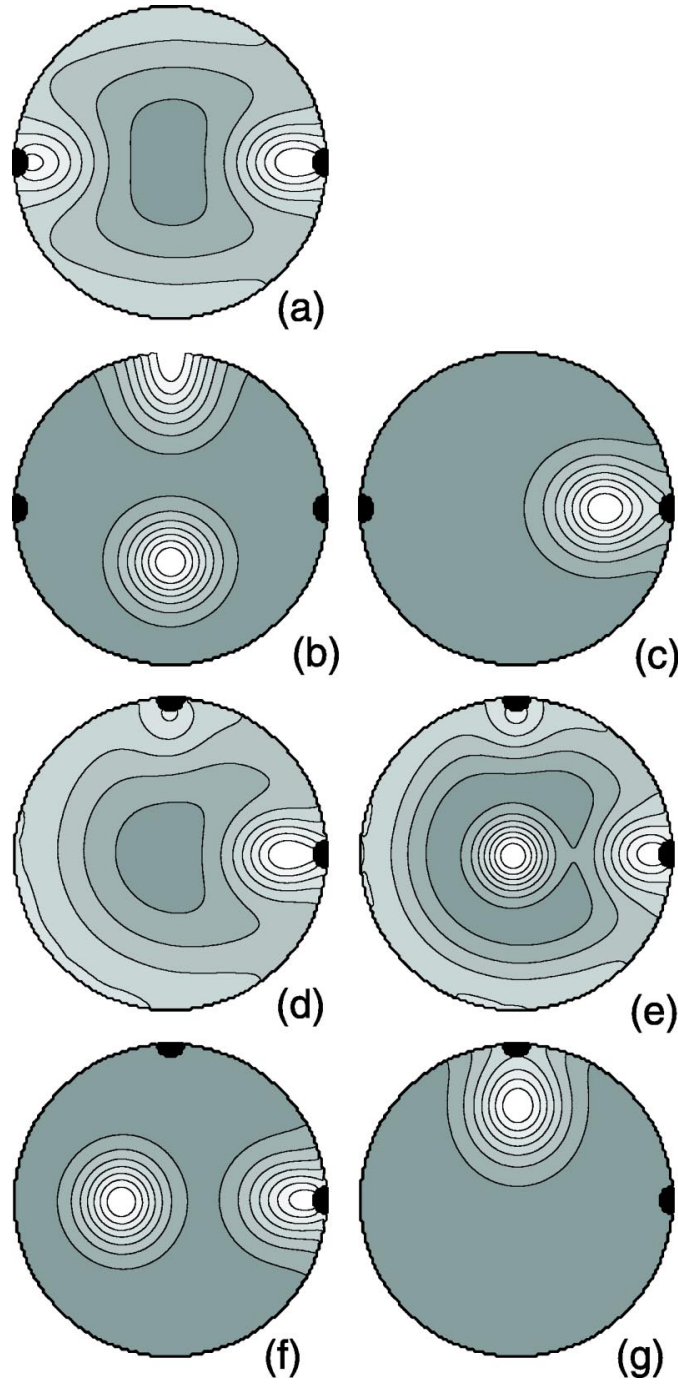

FIG. 3. The Cooper-pair density of the vortex configuration during (a) the $L=0 \rightarrow 2$, (b) the $L=2 \rightarrow 1$, and (c) the $L=1 \rightarrow 0$ transition in sample (iii) of Fig. 1, and (d) the $L=0 \rightarrow 1$, (e) the $L=1$ $\rightarrow 2$, (f) the $L=2 \rightarrow 1$, and (g) the $L=1 \rightarrow 0$ transition in sample (iv) of Fig. 1. High (low) Cooper-pair density is given by dark (white) regions.

creases and expulsion field increases, when surface defects are introduced. The number of vortices entering during the same transition can be influenced by the presence of surface defects. Also, the entry or exit positions of the vortices depend on the presence of surface defects. However, all these conclusions partially depend on the size of the sample and the size of the defect. To illustrate this, we calculated in Fig. 4 the free energy with increasing field for the same sample configurations as above, but now we took the disk radius $R$ $=3.0 \xi$, while we kept the defect radius the same, i.e., $R_{\text {defect }}=0.5 \xi$. In a perfect sample with no surface defects (see the thick solid curve), the vortex state changes at the first transition from the Meissner state to the two-vortex state, just as in the case of the larger sample. For one surface defect, the state transits again from the Meissner state to the $L=1$ state (see the thin solid curve). When we add a second defect opposite to the first one [sample (iii)], the situation is

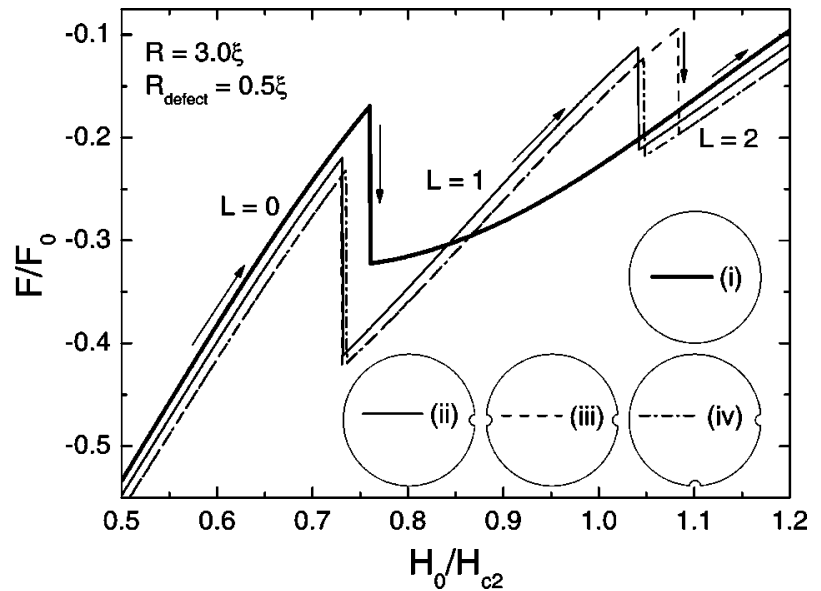

FIG. 4. The free energy as a function of increasing magnetic field for a disk with radius $R=3.0 \xi$ with (i) no surface defects, (ii) one surface defect with radius $R_{\text {defect }}=0.5 \xi$, and (iii), (iv) two surface defects.

different from the larger sample case. Now, only one vortex enters during the first transition (see the dashed curve). The reason is probably that the repulsion between the two vortices is too strong to penetrate together, because they are too close to each other. For sample (iv) we find a similar behavior as for larger samples. Only one vortex enters during the first transition (see the dashed-dotted curve in Fig. 4). Notice further that the free energy decreases when the number of defects increases, which is due to the decrease in the effective superconducting area.

So far, we have investigated the penetration and expulsion of vortices at low fields, when only a few vortices are present in the disk. Next, we will study the effect of a surface defect on the vortex penetration and expulsion at higher fields and vorticities. Figure 5 shows the free energy as a function of the magnetic field for a disk with radius $R=6.0 \xi$ with no surface defects (dashed curves) and one surface defect with

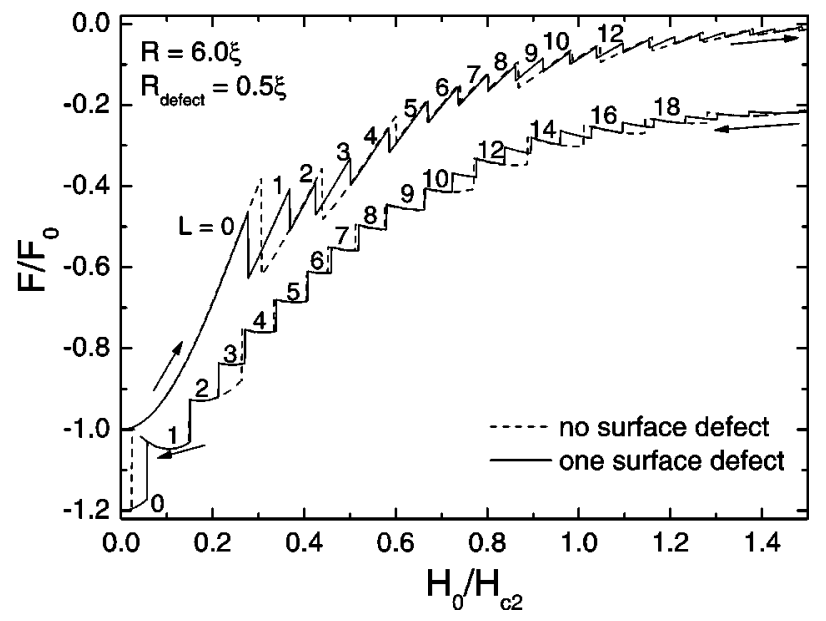

FIG. 5. The free energy as a function of the magnetic field for a disk with radius $R=6.0 \xi$ with no surface defects (dashed curves) and one surface defect with radius $R_{\text {defect }}=0.5 \xi$ (solid curve) when increasing (upper curves) and decreasing (lower curves, shifted over $-0.2 F_{0}$ ) the magnetic field. 


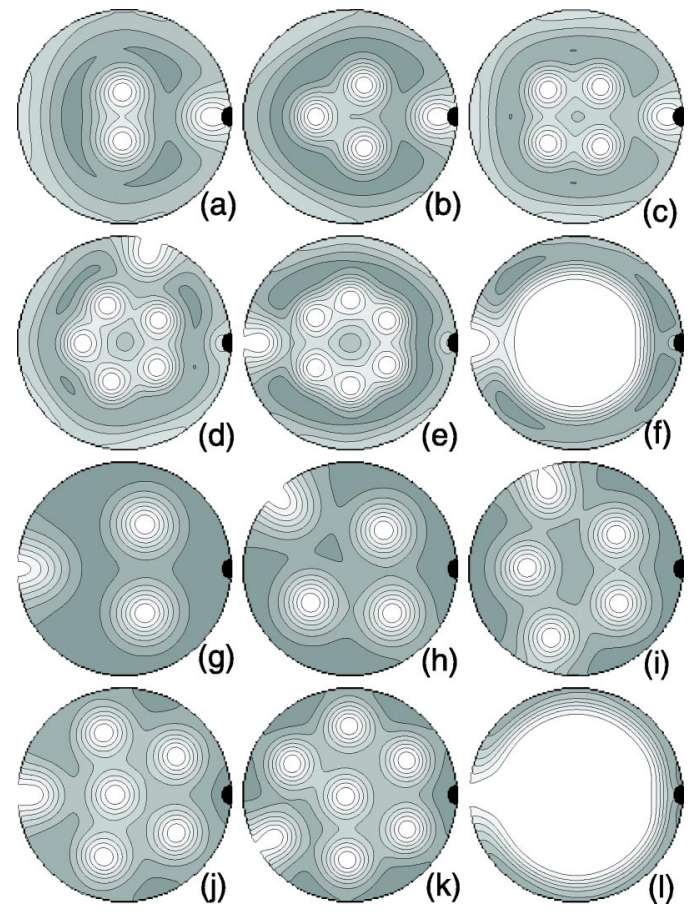

FIG. 6. The Cooper-pair density of the vortex configuration in a disk with radius $R=6.0 \xi$ with one surface defect with radius $R_{\text {defect }}=0.5 \xi$ during the $L \rightarrow L+1$ transition for (a) $L=2$, (b) $L=3$, (c) $L=4$, (d) $L=5$, (e) $L=6$, and (f) $L=12$, and during the $L \rightarrow L$ - 1 transition for (g) $L=3$, (h) $L=4$, (i) $L=5$, (j) $L=6$, (k) $L=7$, and (1) $L=18$. High (low) Cooper-pair density is given by dark (white) regions.

radius $R_{\text {defect }}=0.5 \xi$ (solid curve). By introducing a surface defect, the penetration fields decrease and the vorticity changes always by 1 at the transitions. When decreasing the magnetic field, the expulsion fields increase due to the surface defect and the vortices leave the sample one by one. Notice that in the perfect disk two vortices may enter or leave simultaneously.

Next we investigate the vortex entry and exit positions at higher fields. Do the vortices penetrate or exit the sample through the defect? Figure 6 shows the vortex entry and exit positions in the disk with a circular defect of Fig. 5. Figures 6(a)-6(f) show the entry positions for the $L \rightarrow L+1$ transitions with $L=2,3,4,5,6$, and 12 . For $L=2,3,4$ the vortices enter through the defect, just as we found in Figs. 2(a) and 2(b) for the $L=0 \rightarrow 1$ and the $L=1 \rightarrow 2$ transition. On the other hand, the vortex does not enter through the defect during the $L \rightarrow L+1$ transition when $L \geqslant 5$. The $L=5 \rightarrow 6$ transition is shown in Fig. 6(d). The vortex does not enter through the defect, but also not at the position opposite to the defect. For $L \geqslant 6$ the vortex always enters the disk at the position opposite to the defect, regardless of the fact of whether the initial state is a multivortex or a giant vortex state. Figure 6(e) shows the $L=6 \rightarrow 7$ transition where the initial state is a multivortex state and Fig. 6(f) the $L=12 \rightarrow 13$ transition where the $L=12$ state is a giant vortex state.

With decreasing field, the vortex leaves the sample through the defect during the $L=1 \rightarrow 0$ transition and not through the defect during the $L=2 \rightarrow 1$ transition [see Figs. 2(c) and 2(d)]. At higher fields, we find that the vortex exit never happens through the defect. The reason is that in the initial state the vortices are locked by the defect in such a way that the defect is always exactly situated in between two vortices due to the repulsion with the external magnetic field. With decreasing field, the vortex lattice does not rotate (because it is fixed by the defect) and one vortex just moves in radial direction towards the sample boundary where it leaves the sample. This can be seen from Figs. 6(g)-6(l) where the exit positions are shown for the $L \rightarrow L-1$ transitions with $L$ $=3,4,5,6,7$, and 18 .

\section{B. Surface defects in superconducting thin squares and rectangles}

Next, we consider square superconductors and we investigate the effect of the surface defect position with respect to the corners. Figures 7(a) and 7(b) show the free energy as a function of increasing and decreasing magnetic field for a square superconductor with sides equal to $W=6 \xi$ without a defect (solid curves), with a surface defect in the middle of one of the sides (dashed-dotted curves) and with a surface defect in a corner of the square sample (dashed curves). All the defects are circular and have a radius equal to $R_{\text {defect }}$ $=0.5 \xi$. For the case without surface defects the vorticity in such a square with side $W=6 \xi$ changes by one at the first transition field $H_{0} / H_{c 2}=0.612$, i.e., the state transits from $L$ $=0$ to $L=1$ [see the solid curve in Fig. 7(a)]. By introducing a defect in the middle of one of the sides the first penetration field, where one vortex enters, decreases to $H_{0} / H_{c 2}=0.588$. A defect on the corner has (almost) no effect on the penetration field. The vortex enters through the middle of a side (see also below) and this is too far away from the corner to feel the effect of a defect there. The expulsion of the vortices occurs one by one [see Fig. 7(b)]. A defect in one of the corners increases slightly the expulsion field, while a surface defect in the middle of a side increases the expulsion field more significantly. Figures 7(c) and 7(d) show the same as Figs. 7(a) and 7(b), but now for a square with sides equal to $W=12 \xi$. In such larger squares several vortices can penetrate simultaneously [see Fig. 7(c)]. For the square without surface defects, two vortices enter at the first transition field, like in the circular disk case. Here, of course, a defect in the corner does again not influence the transition, because it is even further away from the penetration position of the vortices as it was for smaller squares. On the other hand, a defect in the middle of one of the sides decreases the first penetration field substantially and also changes the number of vortices entering the superconductor. The vortices enter one by one, i.e., the simultaneous penetration of more vortices is no longer found, because the defect introduces a preferential nucleation site for vortex entry. With decreasing field, the vortices leave one by one in all cases [Fig. 7(d)].

Next we focus on the position of the vortex entry and exit in the systems of Figs. 7(c) and 7(d), i.e., a superconducting square with sides equal to $W=12 \xi$ with no defect, a circular defect with radius $R_{\text {defect }}=0.5 \xi$ in one of the corners, and such a defect in the middle of one of the sides. Figure 8 gives the contour plots of the Cooper-pair density for the vortex 

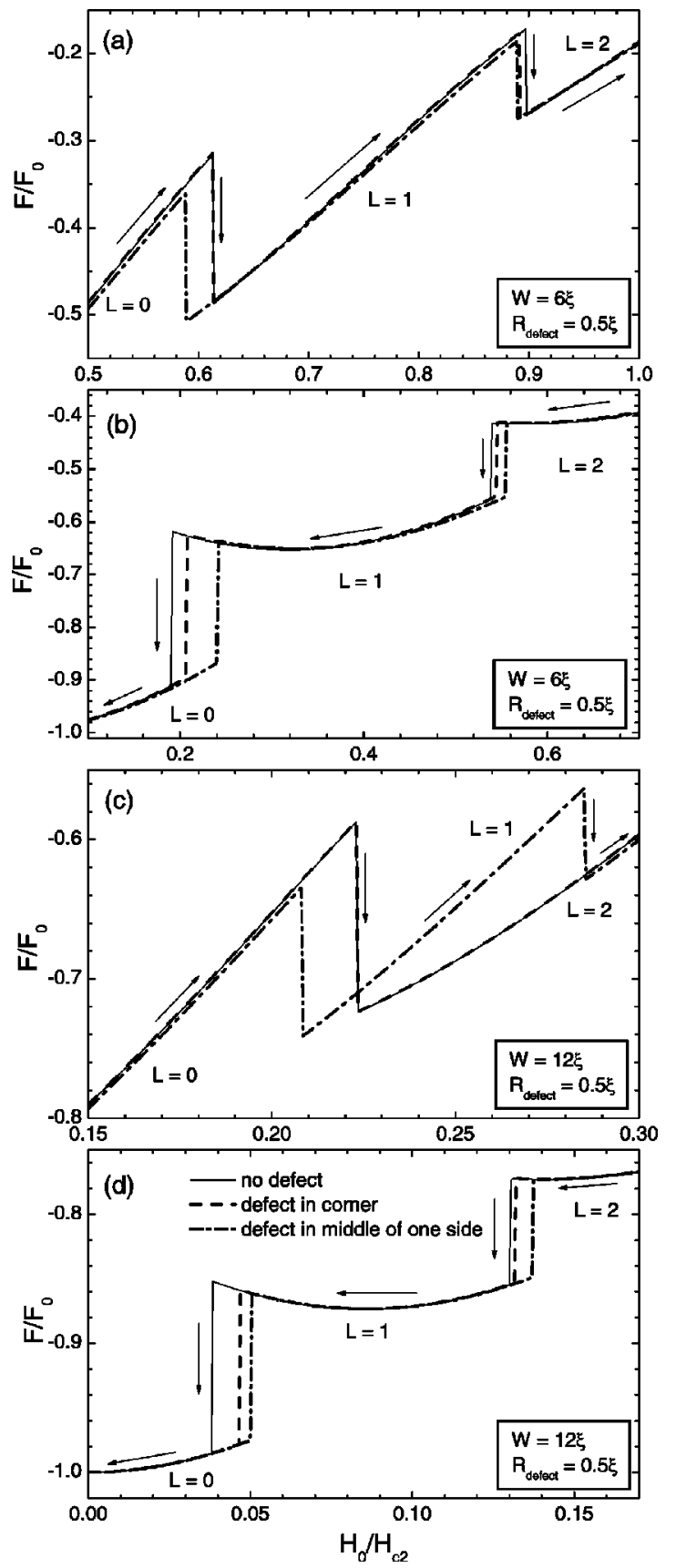

FIG. 7. The free energy as a function of increasing and decreasing magnetic field for (a),(b) a square with size $W=6 \xi$ and (c), (d) a square with size $W=12 \xi$ with no surface defect (solid curve), a surface defect in the corner of the square (dashed curve), and one in the middle of one side of the square (dashed-dotted curve). The surface defect has radius $R_{\text {defect }}=0.5 \xi$.

states during the vortex penetration or expulsion in such a squares. In a perfect square two vortices enter opposite to each other during the first transition [see Fig. 8(a)]. When a surface defect is introduced in the middle of one of the sides, only one vortex enters at the first penetration field [see Fig. 7(c)]. From Fig. 8(b) it is clear that this vortex enters through the defect. Also the second vortex enters through the defect at the next penetration field [Fig. 8(c)]. Notice that the stable vortex configuration for $L=2$ is a multivortex state where the
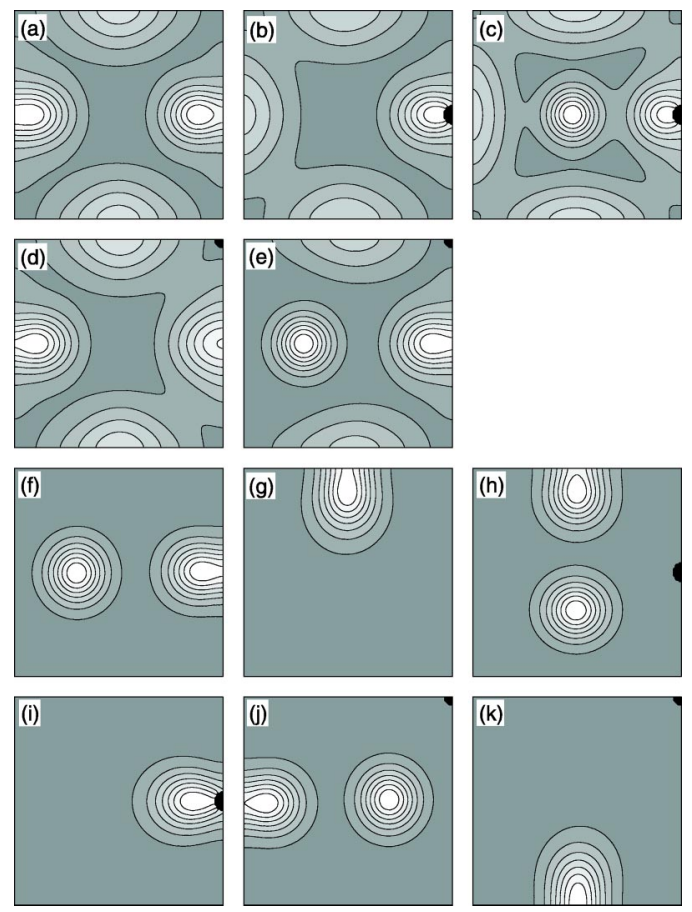

FIG. 8. Contour plots of the Cooper-pair density of the vortex state during the vortex penetration or expulsion in a square with width $W=12 \xi$ and for defects with radius $R_{\text {defect }}=0.5 \xi$. (a) The $L$ $=0 \rightarrow 2$ transition in a perfect square sample, (b) the $L=0 \rightarrow 1$, and (c) the $L=1 \rightarrow 2$ transition in a square with a defect in the middle of one of the sides, (d), (e) the $L=0 \rightarrow 2$ transition in a square with a defect in one of the corners, (f) the $L=2 \rightarrow 1$ and (g) the $L=1 \rightarrow 0$ transition in a perfect square, (h) the $L=2 \rightarrow 1$ and (i) the $L=1$ $\rightarrow 0$ transition in a square with a defect in the middle of one of the sides, (j) the $L=2 \rightarrow 1$, and (k) the $L=1 \rightarrow 0$ transition in a square with a defect in one of the corners. High (low) Cooper-pair density is given by dark (white) regions.

two vortices are situated along one of the two diagonals. So, after the penetration of the second vortex, the vortex molecule will rotate until the state stabilizes with two vortices along one of the two diagonals. The two possible states with vortices along the diagonals have exactly the same free energy, because the distances from the vortices to the defect are the same in both cases. For a defect in the corner, two vortices enter at the same penetration field, i.e., $H_{0} / H_{c 2}=0.223$ through the middle of two opposite sides [see Figs. 8(d) and 8(e)]. Notice that the two figures [see Figs. 8(d) and 8(e)] may suggest that the two vortices do not penetrate simultaneously, i.e., at the same magnetic field. This is not the case. The two vortices do penetrate at the same magnetic field $H_{0} / H_{c 2}=0.223$ as is clear from Fig. $7(\mathrm{c})$, but not at the same iteration step in our numerical process.

Figures 8(f) and 8(g) give the expulsion of the vortices from the perfect square. The last but one vortex leaves through the center of one of the sides [Fig. 8(f)]. In the stable two-vortex state, the two vortices are situated along the diagonal. During the transition, the two vortices first rotate over $\pi / 4$ and then one of the vortices leaves through the middle of one of the sides. Also, during the $L=1 \rightarrow 0$ transition the vortex leaves the superconductor through the center of one of the sides [Fig. $8(\mathrm{~g})]$. When a defect is introduced in 


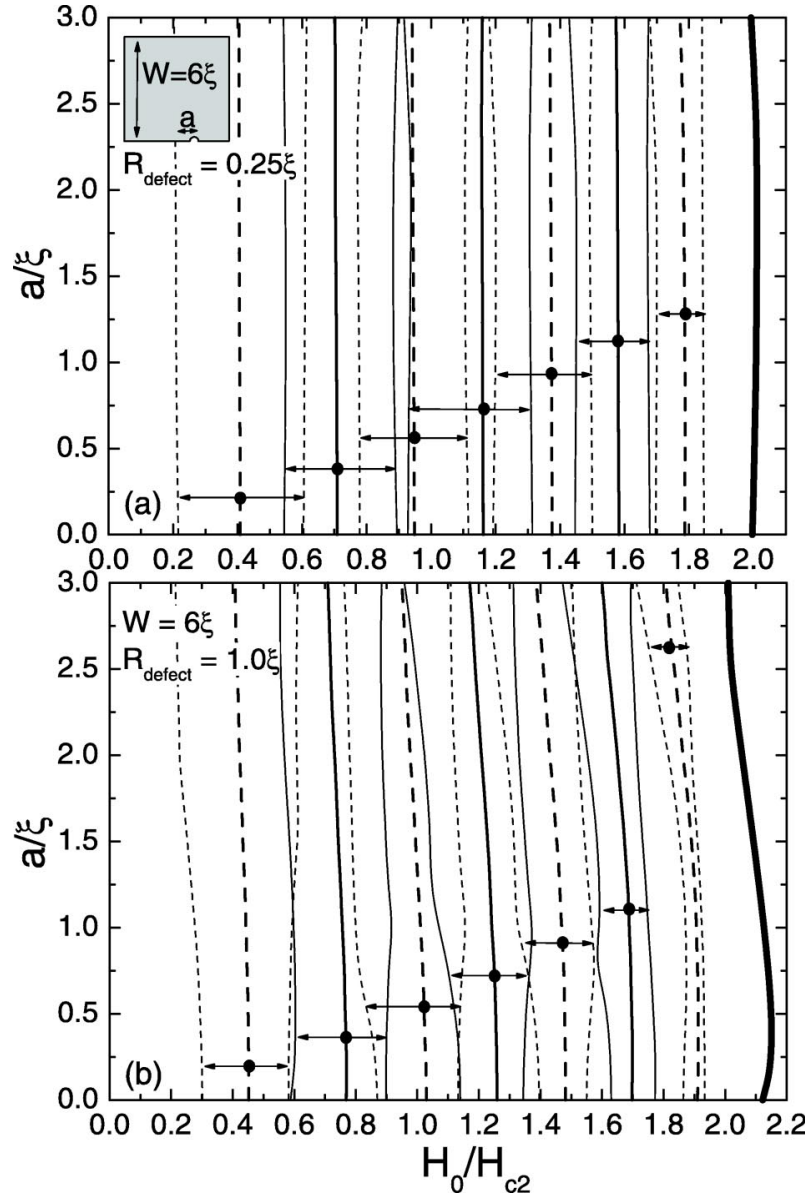

FIG. 9. $a-H_{0}$-phase diagram for a square with $W=6 \xi$, where $a$ is the displacement of the defect from the center of the side and $H_{0}$ the applied magnetic field. The thick curves are the ground state transitions. The thinner curve on the right (left) side from this curve is the penetration (expulsion) field. The $L \leftrightarrow L+1$ transitions are given by solid curves (dashed curves) when $L$ is odd (even). The radius of the circular defect is $R_{\text {defect }}=0.25 \xi$ in (a) and $R_{\text {defect }}$ $=1.0 \xi$ in $(\mathrm{b})$.

the middle of one of the sides the last but one vortex leaves through the center of a side [Fig. 8(h)], and the last vortex leaves through the defect [Fig. 8(i)]. For a defect in the corner, the two vortices leave through the center of a side [Figs. $8(\mathrm{j})$ and $8(\mathrm{k})$ ] and not through the defect.

Now, we will examine in more detail the effect of the surface defect position for a square superconductor with sides equal to $W=6 \xi$. In Fig. 9 phase diagrams are given where $a$ is the distance between the middle of the side and the center of the defect. $a$ is varied from $a=0$, i.e., the middle of the side, to $a=3 \xi$, i.e., the corner of the square. The thick curves in the figures indicate the ground state transitions. The thinner curve on its left side indicate the expulsion field, the one on its right side is the penetration field. The very thick solid curve at the right side of the figure is the superconducting/normal transition field. In an effort to make the figure more clear we used solid curves for $L \leftrightarrow L+1$ transitions when $L$ is odd and dashed curves when $L$ is even. We also add arrows that indicate which penetration and expulsion field belongs to which transition. In Fig. 9(a) we con-
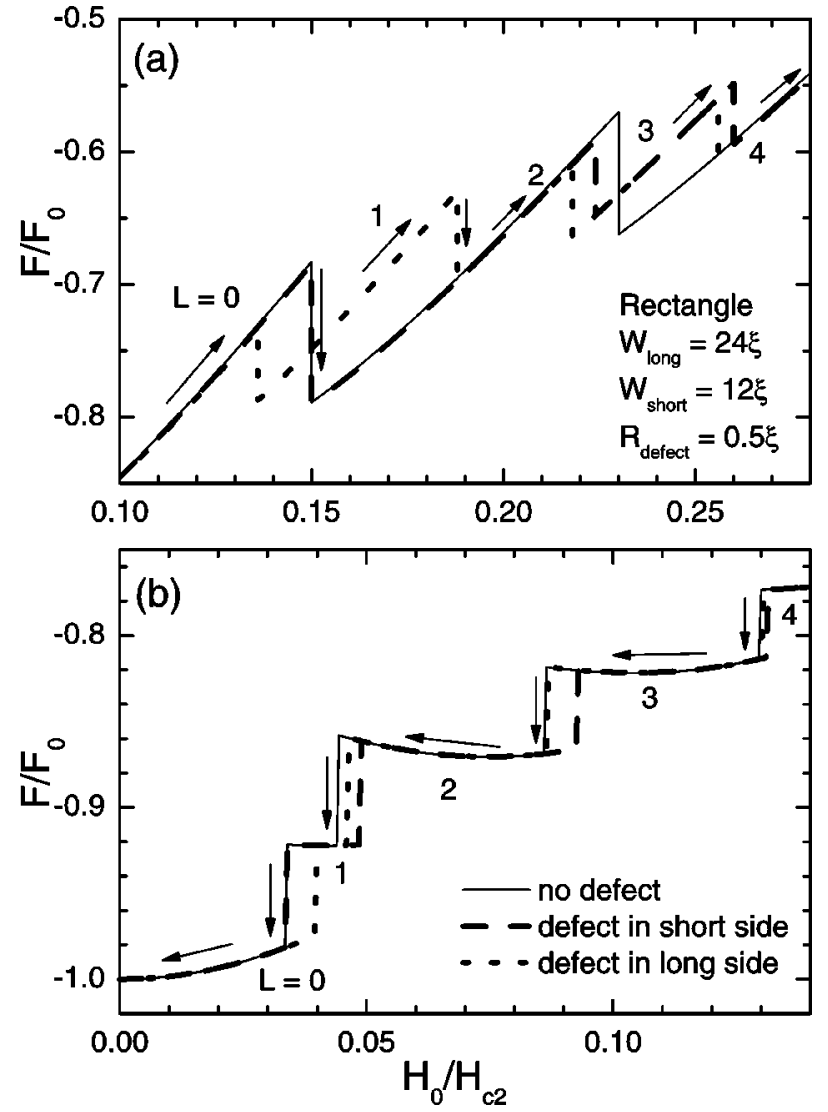

FIG. 10. The free energy as a function of (a) the increasing magnetic field and (b) the decreasing magnetic field for a superconducting rectangle with sides equal to $12 \xi$ and $24 \xi$ with no defect (solid curve), with a defect at the middle of the short side (dashed curve) and one in the middle of the long side (dotted curve).

sidered a defect with radius $R_{\text {defect }}=0.25 \xi$, while in Fig. 9(b) the defect radius equals $R_{\text {defect }}=1.0 \xi$. From Fig. 9(a) we can conclude that the position of a small defect along the surface has only a small effect on the ground state transition fields. The effect on the expulsion field and the penetration field is a little bit larger but also not very striking. Also the superconducting/normal transition seems to be (almost) insensitive to the position of the defect. For larger defects [see Fig. 9(b)], the influence of the defect position on the transition fields is more pronounced. A clear increase of the size of the hysteresis is found with increasing $a$ and this increase is largest for $a=\xi$.

Notice that the difference between the thermodynamic transition field and the field for vortex penetration, i.e., $H_{\text {penetration }}-H_{\text {thermodynamic }}$, is smaller than the similar one for vortex expulsion, i.e., $H_{\text {thermodynamic }}-H_{\text {expulsion }}$, indicating that the barrier for vortex entry is smaller than the barrier for vortex expulsion. This is the case both for $R_{\text {defect }}=0.25 \xi$ [Fig. 9(a)] and for $R_{\text {defect }}=1.0 \xi$ [Fig. 9(b)].

Next, we will investigate the vortex penetration and expulsion in rectangular samples. Is the vortex entry and exit the same for a surface defect in the middle of the long or the short side? Figures 10(a) and 10(b) show the free energy for increasing and decreasing magnetic field for a superconducting rectangle with no surface defects (solid curve), with a 

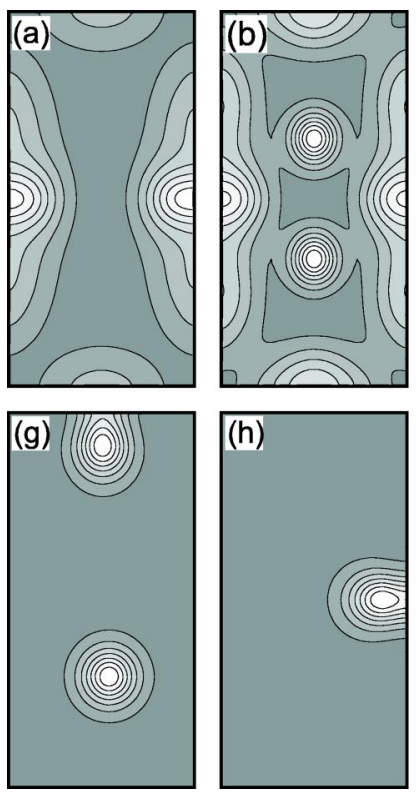
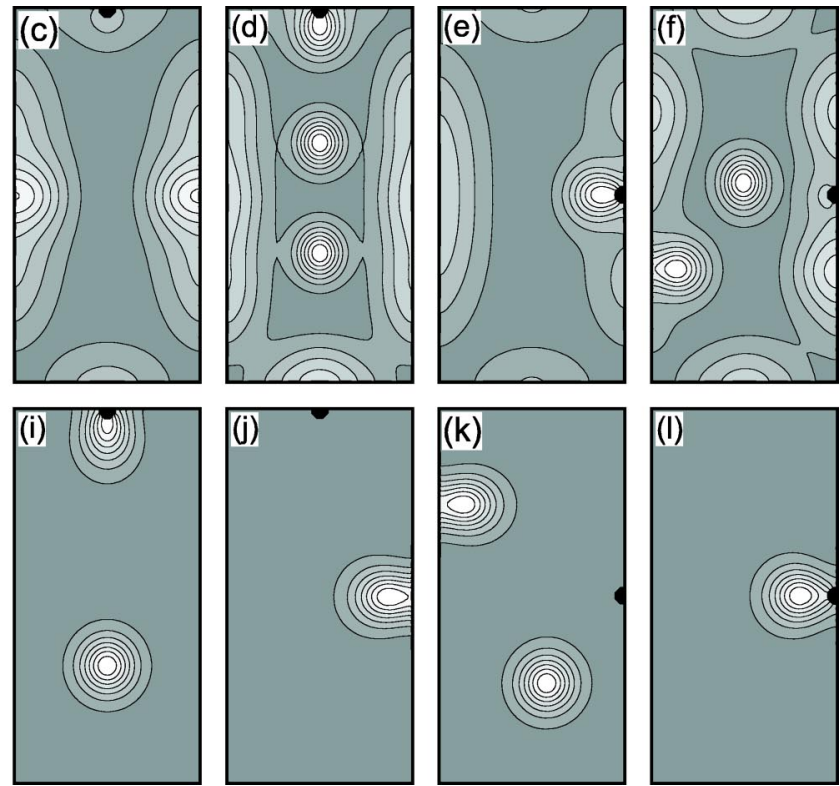

FIG. 11. Contour plots of the Cooper-pair density of the vortex state during the vortex penetration or expulsion in the rectangular samples of Fig. 10. (a),(b) the $L=0 \rightarrow 2$ and the $L=2 \rightarrow 4$ transition in a perfect rectangle, (c), (d) the $L=0 \rightarrow 2$ and the $L=2 \rightarrow 3$ transition in a rectangle with a defect in the short side, (e), (f) the $L=0 \rightarrow 1$ and the $L=1 \rightarrow 2$ transition in a rectangle with a defect in the long side, (g), (h) the $L=2 \rightarrow 1$ and the $L=1 \rightarrow 0$ transition in a perfect rectangle, (i), (j) the $L=2 \rightarrow 1$ and the $L=1 \rightarrow 0$ transition in a rectangle with a defect in the short side, and (k), (1) the $L=2 \rightarrow 1$ and the $L=1 \rightarrow 0$ transition in a rectangle with a defect in the long side. High (low) Cooper-pair density is given by dark (white) regions.

defect in the middle of the long side (dotted curves) and with one in the middle of the short side (dashed curves). The rectangle consists of long sides equal to $24 \xi$ and short sides equal to $12 \xi$. The circular surface defect has radius $R_{\text {defect }}$ $=0.5 \xi$. In Figs. 11(a) $-11(\mathrm{f})$ contour plots of the Cooper-pair density are given for the vortex states during the first two transitions with increasing field for the three sample configurations and in Figs. 11(g)-11(l) the ones during the last two transitions with decreasing field.

In a perfect rectangle, i.e., one without surface defects, the vortices enter at the middle of the long sides two by two. The reason is that the Cooper-pair density along the surface is lowest at this position which makes it the most favorable position for vortices to enter. ${ }^{16}$ Therefore, for the case of a rectangle without surface defects, the vorticity changes by two, i.e., $L=0 \rightarrow 2 \rightarrow 4$ [see the solid curve in Fig. 10(a)]. From Figs. 11(a) and 11(b) it is clear that the first two vortices enter from the middle of the long side at the first transition field, and the next two vortices do the same at the second transition field.

A defect in the middle of the short side does not influence the first transition. The penetration field is the same as the one for the "perfect" rectangle and also the change in vorticity is the same [see Fig. 10(a)]. The defect is too far away from the most favorable entry positions of the vortices, i.e., the middle of the long sides. Figure 11(c) shows the Cooperpair density during the first penetration. It is clear that the first two vortices enter from the middle of the side. The second penetration field decreases by adding a surface defect in the middle of the short side and the vorticity changes now from $L=2$ to $L=3$ [see Fig. 10(a)]. The difference with the first transition (from $L=0$ to $L=2$ ) is that now two vortices are present near the center of the square [see Fig. 11(d)]. The repulsion between the vortices makes that the penetration is a little bit more difficult from the middle of the long side. Now, the surface defect acts as an easier place for penetration and the third vortex enters through the middle of the short side where the defect is located [see Fig. 11(d)].

A defect in the middle of the long side of the rectangle leads to a reduction of the first penetration field [see Fig. 10(a)]. Moreover, only one vortex enters when the Meissner state becomes unstable. The surface defect is introduced at the entering position of the vortices. Therefore, just one vortex enters during the first penetration. This is also shown in Fig. 11(e). Also during the second penetration, only one vortex enters and the vorticity changes from $L=1$ to $L=2$ [see Fig. 10(a)]. Remarkably, the second vortex does not enter through the defect. Due to the presence of the first vortex in the center of the rectangle, four new preferable entry positions for vortices are created along the two long sides as can be seen from Fig. 11(f). The second vortex enters through one of these preferable positions instead of through the defect which is quite close to the vortex and the entering vortex is therefore repelled by this vortex.

With decreasing field the vortices leave the sample one by one for the three considered samples as can be seen from Fig. 10(b). The exit positions of the vortices are clear from Figs. 11(g)-11(1). In a perfect rectangle, the last but one vortex leaves the sample through the center of the short side and the last vortex through the middle of the long side [see Figs. $11(\mathrm{~g})$ and $11(\mathrm{~h})]$. Introducing a small defect in the middle of the short side does not influence the exit positions of the vortices [see Figs. 11(i) and 11(j)]. However, since the defect is introduced at the preferable exit position of the last but one 


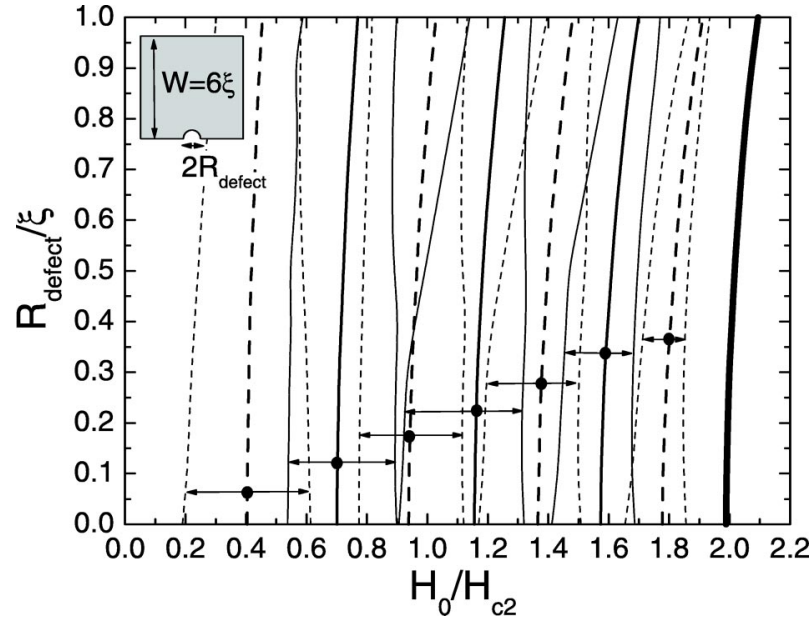

FIG. 12. $R_{\text {defect }}-H_{0}$-phase diagram for a square with $W=6 \xi$, where $R_{\text {defect }}$ is the size of the surface defect in the center of the side and $H_{0}$ the applied magnetic field. The thick curves are the groundstate transitions. The thinner curve on the right (left) side from this curve is the penetration (expulsion) field. The $L \leftrightarrow L+1$ transitions are given by solid curves (dashed curves) when $L$ is odd (even).

vortex in a perfect rectangle, i.e., the middle of the short side, the $L=2 \rightarrow 1$ expulsion moves to higher fields as can be seen from Fig. 10(b). The $L=1 \rightarrow 0$ expulsion field is not influenced since the defect is too far away from the preferable exit position in the middle of the long side. On the other hand, introducing a small defect in the middle of the long side does influence the exit positions of the vortices [see Figs. 11(k) and 11(l)]. Now, the last but one vortex leaves the sample not through the middle of the short side, but prefers to leave it through the long side. The last vortex leaves the sample through the defect which is located at the preferable exit position of the last vortex in a perfect triangle. The influence of adding the defect at this position is that the $L=1$ $\rightarrow 0$ expulsion field moves to higher fields [see Fig. 10(b)].

\section{The effect of the defect size}

We also investigated the influence of the size of the surface defect on the transition fields. Figure 12 shows a phase diagram where $R_{\text {defect }}$ is the radius of the circular surface defect in the middle of one of the sides of the square with sides equal to $6 \xi$. We used the same conventions as in Figs. 9(a) and 9(b). The influence of the defect size is much more pronounced than the one of the defect position and the effect is larger for large $L$ states. All the ground-state transition fields and the superconducting/normal transition field increase with increasing defect size. In general, the expulsion field increases much more than the penetration field with increasing defect size, such that the hysteresis effect decreases. The increase of the $S-N$ field is a consequence of the smaller overall size of the superconductor. It is well known that the superconducting state is stabilized near edges and in small superconductors. ${ }^{17}$

\section{The effect of the finite thickness of the superconductor}

We investigate the effect of the nonzero sample thickness on the penetration and expulsion of vortices. In this case we

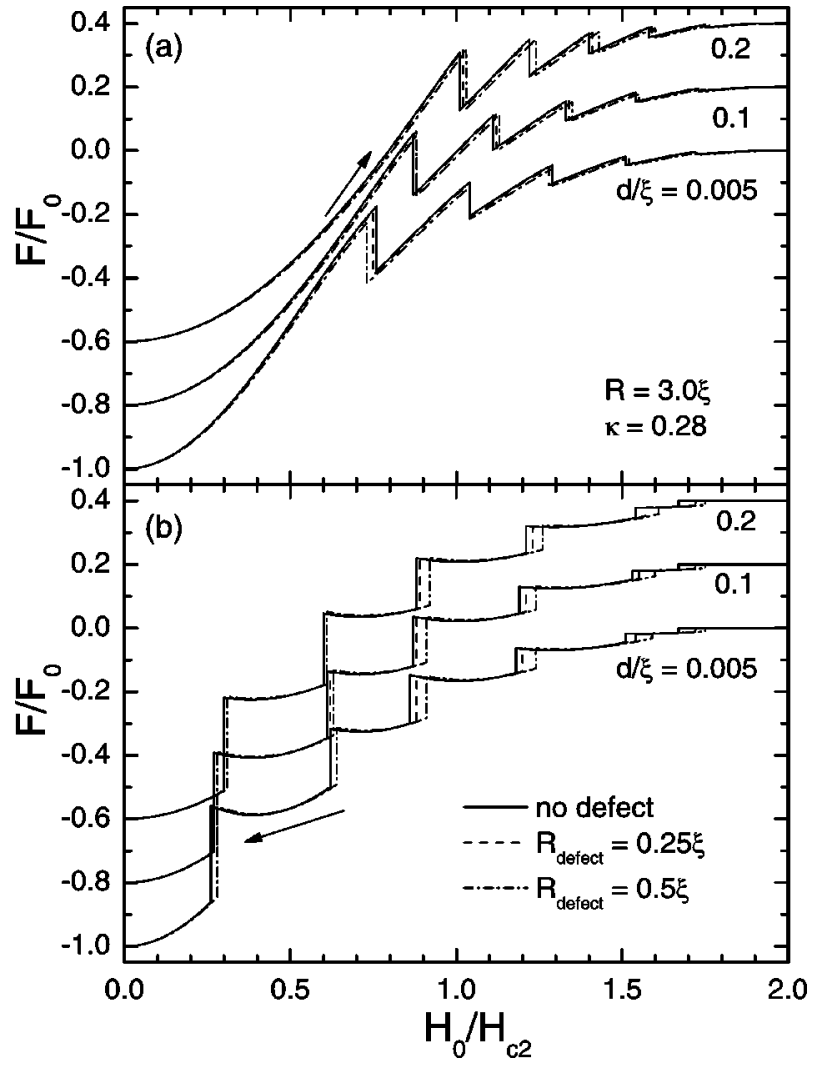

FIG. 13. The free energy for a superconducting disk with no surface defects (solid curves) and with one surface defect with radius $0.25 \xi$ (dashed curves) and $0.5 \xi$ (dashed-dotted curves) as a function of (a) the increasing and (b) the decreasing magnetic field for three values of the sample thickness, i.e., $d=0.005 \xi$ (lower curves), $d=0.1 \xi$ (middle curves, displaced over $0.2 F_{0}$ ), and $d$ $=0.2 \xi$ (upper curves, displaced over $0.4 F_{0}$ ).

can not neglect the second Ginzburg-Landau equation (2) that describes the bending of the magnetic field lines around the superconductor. This means that we have to solve the complete set of nonlinear Ginzburg-Landau equations as described above.

As an example, we consider superconducting disks with radius $R=3.0 \xi$ for Ginzburg-Landau parameter $\kappa=0.28$. Figures 13(a) and 13(b) show the free energy for such a disk with increasing and decreasing field, respectively, for three values of the thickness, i.e., $d=0.005 \xi$ (lower curves), $d$ $=0.1 \xi$ (middle curves, displaced over $0.2 F_{0}$ ), and $d=0.2 \xi$ (upper curves, displaced over $0.4 F_{0}$ ). The free energy for the disk without surface defects is given by solid curves, the one for the disk with one circular surface with radius $R_{\text {defect }}$ $=0.25 \xi$ and $0.5 \xi$ is given by dashed and dashed-dotted curves. With increasing thickness, the hysteresis effect and, hence, the stability region of the metastable states increases in all cases. The penetration field increases significantly, while the change in expulsion field is much smaller. Previously, we found similar behavior in the case of superconducting rings. ${ }^{18}$ The expulsion of the magnetic field is much more efficient in thicker superconductors due to the larger screening currents. From Fig. 13(b) it is clear that the effect of a surface defect on the vortex expulsion does not significantly 
changes with a change in the thickness of the sample. The change in the penetration field is much more pronounced when the thickness of the sample increases and even may lead to a delay of the vortex penetration when a defect is present [see Fig. 13(a)]. Both the expulsion and the penetration field may move to higher fields due to the presence of a defect, but the total hysteresis effect decreases in general due to the defect. Taking into account a finite sample thickness does not significantly change the effect of the defect on the transition fields. Therefore, we expect that the trends shown in our previous results for $d / \xi \ll 1$ stay valid for thin superconductors with a finite thickness.

\section{SUMMARY AND CONCLUSIONS}

In the first part of the present paper we concentrated on extremely thin superconductors, such that only the first GL equation [Eq. (1)] has to be solved and the magnetic field can be assumed to be homogeneous and equal to the external field, also inside the sample. We studied the effect of the number of surface defects on the vortex penetration and expulsion in mesoscopic superconducting disks. The presence of one or more defects decreases the penetration field and enhances the expulsion field in general. With increasing field, the number of vortices simultaneously entering the sample also depends on the presence and the positions of the surface defects. With decreasing field, the vorticity changes always by one unit at the expulsion fields. We found that with increasing field the defect acts as a preferable position for vortices to enter, while with decreasing field the vortices leave the sample in several cases not through the defect. At higher fields, we found that the vortices do not enter or exit through the surface defect, due to the interplay between the vortexvortex repulsion and the vortex-defect interaction.

Next, we investigated the effect of the position of the surface defect on the vortex penetration and expulsion in thin mesoscopic squares. We found that a surface defect in the corner of the square has less influence on the penetration and expulsion fields, than a defect in the middle of the side. This is explained by the fact that the corner is too far away from the preferable entry or exit positions for the vortices. The penetration and exit positions for vortices are not influenced by a defect in the corner, while they are effected by a defect in the middle of a side. It is also found that in the latter case the vortices enter and exit one by one. We constructed phase diagrams which showed the influence of the position of the surface defect in a square sample. For small defects the effect is very weak, while the position of a larger defect plays a more important role. We also studied rectangular samples where we compared the effect of a defect in the middle of the short or the long side of the rectangle. Defects on the long side of the rectangle have a more pronounced effect on the vortex expulsion and penetration, because they act more effectively as nucleation centers for vortex entry and expulsion. However, in both cases the vortex entry and exit positions are influenced by the defects.

We constructed a phase diagram which showed the effect of the defect size on the transition fields. In general, we found that the influence of the defect size on the transition fields is much more pronounced than the influence of the defect position.

In the last part, we investigated the effect of the nonzero sample thickness on the penetration and expulsion of vortices. In this case we solved the complete set of GinzburgLandau equations (1)-(3). We found that a change in the thickness of the sample does not significantly changes our conclusions on the effect of surface defects on vortex penetration and expulsion.

With this study, we showed clearly that the spatial position and the magnetic field for vortex entry and exit can be engineered through the introduction of surface defects in a sample. The size and exact location of these surface defects are important.

\section{ACKNOWLEDGMENTS}

This work was supported by the Flemish Science Foundation (FWO-V1), The Belgian Science policy, and the 21st Century COE Program of MEXT. B. J. Baelus acknowledges support from the Japan Society for the Promotion of Science and FWO-V1.
*Electronic address: ben.baelus@ua.ac.be

${ }^{\dagger}$ Electronic address: kadowaki@ims.tsukuba.ac.jp

Electronic address: francois.peeters@ua.ac.be

${ }^{1}$ V. V. Moshchalkov, L. Gielen, C. Strunk, R. Jonckheere, X. Qiu, C. Van Haesendonck, and Y. Bruynseraede, Nature (London) 373, 319 (1995).

${ }^{2}$ A. K. Geim, I. V. Grigorieva, S. V. Dubonos, J. G. S. Lok, J. C. Maan, A. E. Filippov, and F. M. Peeters, Nature (London) 390, 256 (1997).

${ }^{3}$ V. A. Schweigert and F. M. Peeters, Phys. Rev. B 57, 13817 (1998).

${ }^{4}$ V. A. Schweigert, F. M. Peeters, and P. Singha Deo, Phys. Rev. Lett. 81, 2783 (1998).

${ }^{5}$ A. K. Geim, S. V. Dubonos, J. G. S. Lok, M. Henini, and J. C.
Maan, Nature (London) 396, 144 (1998).

${ }^{6}$ A. K. Geim, S. V. Dubonos, I. V. Grigorieva, K. S. Novoselov, F. M. Peeters, and V. A. Schweigert, Nature (London) 407, 55 (2000).

${ }^{7}$ J. J. Palacios, Phys. Rev. Lett. 84, 1796 (2000).

${ }^{8}$ P. S. Deo, V. A. Schweigert, and F. M. Peeters, Phys. Rev. B 59, 6039 (1999).

${ }^{9}$ F. M. Peeters, B. J. Baelus, and V. A. Schweigert, Physica C 369, 158 (2002).

${ }^{10}$ D. Yu. Vodolazov, Phys. Rev. B 62, 8691 (2000).

${ }^{11}$ D. Y. Vodolazov, I. L. Maksimov, and E. H. Brandt, Physica C 384, 211 (2003)

${ }^{12}$ G. M. Braverman, S. A. Gredeskul, and Y. Avishai, Low Temp. Phys. 25, 614 (1999). 
${ }^{13}$ V. A. Kashurnikov, I. A. Rudnev, and M. V. Zyubin, JETP 94, 377 (2002).

${ }^{14}$ B. J. Baelus and F. M. Peeters, Phys. Rev. B 65, 104515 (2002).

${ }^{15}$ V. A. Schweigert and F. M. Peeters, Phys. Rev. Lett. 83, 2409 (1999).

${ }^{16}$ G. Teniers, L. F. Chibotaru, A. Ceulemans, and V. V. Mosh- chalkov, Europhys. Lett. 63, 296 (2003).

${ }^{17}$ V. A. Schweigert and F. M. Peeters, Phys. Rev. B 60, 3084 (1999).

${ }^{18}$ B. J. Baelus, F. M. Peeters, and V. A. Schweigert, Phys. Rev. B 61, 9734 (2000). 